\title{
ANÁLISIS DE LA PRESENCIA DE ISLAS DE CALOR EN EL CANTÓN RUMIÑAHUI MEDIANTE EL TRATAMIENTO DE IMÁGENES LANDSAT 7 ETM+
}

\author{
ANALYSIS OF THE PRESENCE OF HEAT ISLANDS IN THE RUMIÑAHUI CANTON \\ THROUGH THE TREATMENT OF ETM+ LANDSAT 7 IMAGES
}

LISSETH JAMI, MARÍA BELÉN MORA, OSWALDO PADILLA, EDUARDO KIRBY.

CARRERA DE INGENIERÍA GEOGRÁFICA Y DEL MEDIO AMBIENTE, DEPARTAMENTO DE CIENCIAS DE LA TIERRA Y DE LA CONSTRUCCIÓN, UNIVERSIDAD DE LAS FUERZAS ARMADAS - ESPE. Av. General Rumiñahui s/n, Sangolquí, Ecuador. liseth_dic@hotmail.com, mabelenmorap@gmail.com, ovpadilla@espe.edu.ec, epkirby@espe.edu.ec

Recibido: 02 de agosto 2018 / Aceptado: 20 de diciembre 2018

\section{RESUMEN}

Se considera que en los últimos años la temperatura superficial ha aumentado, debido a factores como cambio de uso de suelo, crecimiento de zonas urbanas y densidad poblacional dando lugar a fenómenos térmicos tales como las islas de calor. Mediante el uso de sensores remotos con banda térmica, es posible determinar las variaciones en los patrones de temperatura superficial, su extensión e intensidad. El cantón Rumiñahui está ubicado en Ecuador, provincia de Pichincha, donde los asentamientos en las zonas urbanas han ido en aumento en los últimos años, es por ello que se hace necesario el análisis de temperatura superficial además de su relación con factores tales como vegetación, agua y construcciones para determinar si estos son incidentes en su variación. Para ello se utilizó dos imágenes Landsat 7 ETM+ la primera correspondiente al 2001 y la segunda al 2016 a partir de las cuales se determinó temperatura superficial e índices tales como NDVI (Índice Diferencial Normalizado de Vegetación), NDWI (Índice Diferencial Normalizado de Agua), NDBI (Índice Diferencial Normalizado de Construcciones) para establecer si existe relación entre ellos mediante un análisis de correlación de variables obteniéndose que estos factores presentan una fuerte correlación con valores que varían entre 0,6 a 0,9 . Finalmente se determinó que las zonas con mayor temperatura se encuentran en el centro de la ciudad de Sangolquí y San Rafael.

Palabras Claves: agua, construcción, vegetación, índices, temperatura.

\begin{abstract}
It is considered that in recent years the surface temperature has increased, this phenomenon may be due to the change in land use, growth of urban areas and population density leading to thermal phenomena such as heat islands. Through the use of remote sensors with thermal band, it is possible to determine the variations in the surface temperature patterns, their extension and intensity. Rumiñahui canton is located in Ecuador, in the province of Pichincha, where settlements in urban areas has been increasing in recent years, which is why it is necessary to analyze surface temperature in addition to its relationship with other factors such as vegetation, water and buildings to determine if these are incidents in the variation thereof. To do this, two Landsat 7 ETM + images were used, the first corresponding to 2001 and the second to 2016, from which surface temperature was determined and indexes such as NDVI (Normalized Vegetation Differential Index), NDWI (Normalized Water Differential Index), NDBI (Normalized Differential Construction Index) to establish if there is a relationship between them by means of a correlation analysis of variables, obtaining that these factors present a strong correlation with values that vary between 0.6 to 0.9 . Finally, it was determined that the zones with the highest temperature are in the center of the city of Sangolquí and San Rafael.
\end{abstract}

Key words: water, building, vegetation, rating, temperature. 


\section{INTRODUCCIÓN}

Los procesos de urbanización y cambio de uso del suelo son constantes, lo que trae una serie de problemas relacionados con el cambio climático, siendo uno de estos, el fenómeno de islas de calor (Moreno \& Serra, 2016).

En los últimos años, el análisis de islas térmicas ha tomado interés de organizaciones enfocadas a estudios ambientales, de la misma manera organismos como el Panel Intergubernamental de Cambio Climático-IPCC (IPCC, 2007) y la Organización Mundial de Meteorología (OMM, 2010) analiza el incremento en la ocurrencia de olas de calor que puede estar vinculada a efectos de cambio climático; durante la mitad del siglo XX y la primera década del XXI, ha tomado interés por parte de comunidad científica encargada de estudios ambientales principalmente por investigadores que se centran en las modificaciones climáticas antropogénicas en el sector urbano, así como también su relación con el dosel urbano, vegetación, hidrografía y atmósfera debido principalmente al impacto ambiental que generan estas islas térmicas. (Carreras \& Martín, 1990); (Moreno M. , 1993); (Stewart, 2011); (Noro \& Lazzarin, 2015).

Para el estudio de estas islas de calor, es necesario una red completa de estaciones hidroclimáticas, radiómetros y sondeos verticales atmosféricos (EPA, 2009), lo cual implica un alto costo de adquisición y mantenimiento de los mismos, por lo que el uso de sensores remotos y la aplicación de técnicas de percepción remota se ha convertido en una opción viable (Preciado \& Aldana, 2011).

El impacto ambiental al darse el fenómeno de islas de calor es evidente, ya que se hace necesario el uso de sistemas de climatización, lo que involucra la calidad del aire por los contaminantes emanados, lo que implica en el bienestar de la población que habita en el sector como en la salud (Tumini, 2010).

Existen numerosas ventajas del uso de sensores remotos para este tipo de estudios, entre ellos se incluye la posibilidad de abarcar grandes extensiones, además de ello se puede realizar análisis complementarios como los obtenidos con los siguientes índices: Índice de Vegetación Diferencial Normalizado (NDVI), Índice de Construcciones Diferencial Normalizado (NDBI) e Índice de Agua Diferencial Normalizado (NDWI) para determinar si estos factores tienen influencia en el comportamiento térmico de la ciudad y los alrededores (Preciado \& Aldana, 2011). La elaboración de mapas de temperatura posibilita la identificación de áreas expuestas a mayor riesgo asociado a eventos extremos de calor y contribuye con el diseño de estrategias de mitigación o atención a la población. Las islas térmicas suelen asociarse con anomalías superficiales de temperatura, por ello es primordial su cuantificación y caracterización (Córdova, 2011).

Existen estudios realizados del fenómeno de islas de calor en las ciudades de Quito, Guayaquil en donde se pudo constatar que las temperaturas más frescas están en lugares 
con cercanía a las áreas verdes; y Cuenca en la cual se llegó a determinar que la temperatura superficial supera a la temperatura ambiente y los puntos de mayor calor superficial corresponden a zonas con espacios de poca y nula vegetación, mayor afluencia vehicular, mayor afluencia de personas y de actividades industriales; en los estudios mencionados se aplicaron diferentes técnicas, de cualquier forma se llegó a conclusiones similares en cuanto a la influencia de áreas urbanas en la temperatura superficial, sin embargo en esta área de estudio se encuentran zonas que no cuentan con las características que presenta un valle principalmente en cuanto a clima; por lo que el desarrollo de esta investigación contribuye a tener una referencia en la presencia de islas de calor en las zonas que se encuentren ubicadas en valles (Palacios, 2013); (Guillen Mena, 2016).

En el 2010 con los Códigos y Leyes del COOTAD en los que se encuentra la obligatoriedad del desarrollo de planes de desarrollo y ordenamiento territorial para los gobiernos autónomos, obligaron al desarrollo del mismo para el cantón Rumiñahui, sin embargo datos del INEC en el mismo año muestran que cerca del $92 \%$ de la población ya se encuentra ubicadas en zonas urbanas por ende existe presión en medio natural y construido ya para ese año, la rápida ocupación del suelo para construcciones ocasionan la formación de cañones profundos que obstruyen el paso del viento aumentando la presencia de islas de calor, una medida de mitigación puede ser realizar un correcto trazado urbano (Palacios et al., 2017), es por lo que el desarrollo de este proyecto aporta como un insumo para el desarrollo de una planificación urbana sostenible del cantón Rumiñahui.

El cantón Rumiñahui se ubica al sureste de la Provincia de Pichincha del Ecuador a cerca de veinte minutos de la ciudad de Quito, se caracteriza por un clima agradable y su temperatura promedio de $17^{\circ} \mathrm{C}$. Este cantón se encuentra ubicado en el Valle de los Chillos y su capital es la ciudad de Sangolquí, su extensión es de $134.15 \mathrm{~km}^{2}$. En el cantón hay un notable crecimiento urbanístico, también se puede encontrar vegetación a lo largo de todo el sector, por lo cual el objetivo de este trabajo es calcular la temperatura superficial para determinar las islas de calor e índices como Diferenciado de Vegetación Normalizada (NDVI) con el fin de realzar las áreas con mayor vigor vegetal, Diferenciado de Construcción Normalizada (NDBI) con el cual se identifica que tan sensible es el área urbanizada en la zona de estudio y Diferenciado de Agua Normalizada (NDWI) que permite identificar la medida de cantidad de agua que posee la vegetación o el nivel de saturación de humedad que posee el suelo (Preciado \& Aldana, 2011); de dos imágenes Landsat 7 ETM+ correspondientes a octubre de 2001 y noviembre de 2016 con resolución espacial de $60 \mathrm{~m}$ de la zona de estudio para así determinar si existe cambio en la cobertura del suelo ya sea de aumento o disminución de vegetación, agua y construcciones y si los índices mencionados tienen influencia en la presencia de islas de calor mediante una análisis de correlación. 


\section{MÉTODO}

\section{CÁLCULO DE TEMPERATURA}

Para la realización de este trabajo se seleccionaron dos imágenes LANDSAT 7 ETM+, correspondientes a octubre-2001 y noviembre-2016 las cuales fueron obtenidas de la página web del Servicio Geológico de los Estados Unidos (USGS).

Las imágenes seleccionadas, al ser de nivel L1, ya incorporan correcciones geométricas, por lo cual se llevó a un proceso de corrección radiométrica con el objeto de reducir los efectos atmosféricos logrando de esta manera corregir la nubosidad y otros factores que afectan la interpretación y el análisis de las mismas.

Los valores de temperatura superficial se obtienen de la banda 6 del infrarrojo térmico $(10,44$ a $12,42 \mu \mathrm{m})$ en modo de alta ganancia (6-2 high gain), que tiene una resolución espacial de $60 \mathrm{~m}$. Para ello es necesario convertir los números digitales de la banda térmica en radiancia espectral en el techo de la atmósfera utilizando (1) dada por (Barsi, 2005):

$$
\mathrm{L}_{\mathrm{TOA}}=\tau^{*} \varepsilon_{\mathrm{iv}} * \mathrm{~L}^{\mathrm{T}}+\mathrm{L}_{\mathrm{U}}+\tau *\left(1-\varepsilon_{\mathrm{iv}}\right) \mathrm{L}_{(\mathrm{d})}
$$

Donde: es la transmisividad atmosférica, $\mathrm{L}_{\mathrm{T}}$ es la banda de radiancias calibradas, es la emisividad, es la radiancia ascendente, es la radiancia descendente, $\mathrm{L}_{\mathrm{TOA}}$ es determinado por la radiancia espectral en el techo de la atmósfera.

Caselles, 2001 muestra la emisividad tabulada en ciertas coberturas y por longitudes de onda, estos se pueden visualizar en la Tabla 1, donde $\varepsilon_{\text {ig }}$ y corresponde a la emisividad del suelo y vegetación respectivamente, se determina que en la región de 10,5 - 12,5 () la ecuación operativa propuesta en su investigación, proporciona la emisividad sin desviación sistemática y con un error de $\pm 0,011$, por lo que se ha tomado el valor de emisividad de 0,985 , valor que fue obtenido mediante la media de los valores propuestos tomando en cuenta las coberturas que se pueden presentar en la zona de estudio.

Tabla 1. Valores de emisividad según regiones espectrales (Caselles, 2001).

\begin{tabular}{|c|c|c|c|c|}
\hline & $\mathbf{8 - 9} \boldsymbol{\mu m}$ & $\mathbf{1 0 , 5}-\mathbf{1 1}, \mathbf{5} \boldsymbol{\mu m}$ & $\mathbf{1 1}, \mathbf{5}-\mathbf{1 2}, \mathbf{5} \boldsymbol{\mu m}$ & $\mathbf{1 0 , 5}-\mathbf{1 2}, \mathbf{5} \boldsymbol{\mu m}$ \\
\hline$\varepsilon_{i g}$ & $0,9 \pm 0,06$ & $0,95 \pm 0,02$ & $0,97 \pm 0,01$ & $0,96 \pm 0,014$ \\
\hline$\varepsilon_{i v}$ & $0,985 \pm 0,005$ & $0,985 \pm 0,007$ & $0,985 \pm 0,008$ & $0,985 \pm 0,005$ \\
\hline$\left.<d \varepsilon_{i}\right\rangle$ & $0,04 \pm 0,03$ & $0,022 \pm 0,014$ & $0,013 \pm 0,009$ & $0,017 \pm 0,011$ \\
\hline
\end{tabular}

Los parámetros tales como transmisividad $(\tau)$, radiancia ascendente $\left(\mathrm{L}_{\mathrm{U}}\right)$ y descendente $\left(\mathrm{L}_{\mathrm{d}}\right)$ indicados en la tabla 2, se obtuvieron en la página de corrección atmosférica de la NASA: https://atmcorr.gsfc.nasa.gov/, en la cual es necesario llenar campos como la fecha de toma de la imagen, la hora, la latitud y longitud media, la estación correspondiente a la fecha de toma de la imagen, el sensor y el tipo de interpolación la cual se escogió el uso 
de perfil atmosférico para una longitud y latitud dada, para que así se interpole en torno a las cuatro esquinas de la imagen (NASA, 2017), de lo cual se obtuvieron los parámetros indicados en la Tabla 2:

Tabla 2. Coeficientes de corrección atmosférica.

\begin{tabular}{|l|c|c|}
\hline & Imagen (3/11/2001) & Imagen (27/10/2016) \\
\hline$\tau$ & 0,55 & 0,43 \\
\hline $\mathrm{L}_{\mathrm{U}}$ & 3,36 & 4,54 \\
\hline $\mathrm{L}_{\mathrm{d}}$ & 5,11 & 6,48 \\
\hline
\end{tabular}

Estos valores de radiancia de la banda térmica fueron transformados a temperatura de brillo de superficie usando las constantes de calibración térmica de Landsat 7 ETM+ obtenidas de los metadatos de la imagen mediante (2) dada por (Chuvieco, 2010):

$$
\mathrm{T}_{\mathrm{b}}=\frac{\mathrm{K}_{1}}{\ln \left(\frac{\mathrm{K}_{2}}{\mathrm{~L}}+1\right)}
$$

Donde: $\mathrm{L}$ es el valor de radiancia [W / $\left.\left(\mathrm{m}^{2} \mathrm{sr} \mu \mathrm{m}\right)\right], \mathrm{K}_{1}$ y $\mathrm{K}_{2}$ son las constantes de calibración $\left[\mathrm{W} /\left(\mathrm{m}^{2} \mathrm{sr} \mu \mathrm{m}\right)\right], \mathrm{T}_{\mathrm{b}}$ es determinado por la temperatura de brillo superficial [K] del área en cuestión.

A continuación, se procede al cálculo de la temperatura superficial para la cual se tuvo en cuenta la corrección de la emisividad espectral. Esta se calculó mediante el uso de (3) proporcionada por (Chuvieco, 2010):

$$
\begin{aligned}
\mathrm{T}_{\mathrm{s}} & =\frac{\mathrm{T}_{\mathrm{b}}}{1+\left(\frac{\lambda \mathrm{T}_{\mathrm{b}}}{\rho}\right) * \ln \mathrm{e}} \\
\rho & =\frac{\mathrm{h} * \mathrm{c}}{\mathrm{k}}
\end{aligned}
$$

Donde: $T_{b}$ es la temperatura de brillo superficial $(K), \lambda$ es el centro de la longitud de onda $(\mu \mathrm{m})$, la constante $\rho=13608,69565(\mu \mathrm{m} / \mathrm{K})$ se la obtuvo a partir de (4), teniendo en cuenta a como la constante de Planck $\left(6,26^{*} 10-34 \mathrm{Js}\right)$, c velocidad de la luz $\left(3 \times 10^{8} \mathrm{~m} / \mathrm{s}\right)$ y k la constante de Boltzman $\left(1,38 \times 10^{-23} \mathrm{JK}^{-1}\right)$; e representa la superficie de emisividad, $\mathrm{T}_{\mathrm{s}}$ es determinada por la temperatura superficial $(\mathrm{K})$ del área en cuestión. 


\section{CÁLCULO DE ÍNDICES}

Para realizar el cálculo de los diferentes índices es necesario realizar una corrección radiométrica de reflectancias a las imágenes, para el proceso mencionado se ha utilizado el método de mínimos del histograma.

Índice De Vegetación Diferencial Normalizado (NDVI)

El Índice Normalizado de Vegetación (NDVI), es un cociente empleado para discriminar masas vegetales calculado usando (5) dada por (Marini, 2008):

$N D V I=\frac{N I R-R}{N I R+R}$

Donde: NIR es la Banda del infrarrojo cercano, es la Banda de visible rojo, es determinado por el Î́ndice de Vegetación Normalizado del área en cuestión.

Índice De Agua Diferencial Normalizado NDWI

El Índice de Agua de Diferencia Normalizada (NDWI) es sensible a los cambios en el contenido de agua de la vegetación y se utiliza como una medida de la cantidad de agua que posee la vegetación o el nivel de saturación de humedad que posee el suelo calculado mediante (6) dada por (SNIA, 2016).

$N D W I=\frac{N I R-S W I R_{1}}{S N I R-S W I R_{1}}$

Donde: NIR es la Banda del infrarrojo cercano, $\mathrm{SWIR}_{1}$ es la banda del Infrarrojo de onda corta.

Índice De Construcción Diferencial Normalizado NDBI

El Índice Diferenciado de Construcciones Normalizada (NDBI) destaca las áreas urbanas con mayor reflectancia en la región infrarroja de onda corta (SWIR), en comparación con la región cercana al infrarrojo (NIR), se calculó mediante el uso de (7) dada por (Gao, 1996).

$$
N D B I=\frac{S W I R_{2}-N I R}{S W I R_{2}+N I R}
$$


Donde: $\mathrm{SWIR}_{2}$ es la banda del infrarrojo de onda corta, NIR banda del infrarrojo cercano, NDBI es determinado por el Índice Diferenciado de Construcciones Normalizada del área en cuestión.

\section{ANÁLISIS ESTADÍSTICO}

Como parte de estudio se realizó un análisis estadístico de correlación entre las variables temperatura y los diferentes índices obtenidos para determinar si estos influyen en su comportamiento.

Para ello se partió de los datos obtenidos de los diferentes procesos y se analizó si dentro de estos existen valores atípicos que puedan influenciar en los resultados y se procedió a eliminarlos mediante un análisis por cuartiles. Finalmente se obtuvo el coeficiente de correlación lineal entre las diferentes variables utilizando (8) dada por (Montgomery , 2011).

$$
r=\frac{\sum(x-\bar{x})(y-y)}{(n-1) S_{x} S_{y}}
$$

Donde: $\mathrm{x}, \mathrm{y}$ representan las variables, son las medias de cada variable, $\mathrm{x}^{-}, \mathrm{y}^{-}$es el tamaño de la muestra y $\mathrm{S}_{\mathrm{x}}, \mathrm{S}_{\mathrm{y}}$ son las desviaciones estándar de cada muestra.

\section{ANÁLISIS DE RESULTADOS}

\section{CUANTIFICACIÓN DE LA TEMPERATURA SUPERFICIAL}

Se evidencia un aumento de temperatura superficial en varias zonas del cantón durante los años tomados para el estudio. En la imagen del año 2016, la isla formada en la parte urbana del cantón Rumiñahui no muestra el patrón clásico concéntrico, ésta presenta un carácter geométrico en la ciudad de Sangolquí y San Rafael, mientras que en las poblaciones de Amaguaña y Cotogchoa de cierta forma si es concéntrica como se muestra en la Figura 1, las temperaturas oscilan entre 25 y $28^{\circ} \mathrm{C}$. Se nota que los espacios densamente ocupados y por ende con poca vegetación tienen una mayor temperatura superficial que las zonas de la periferia urbana.

Las áreas con una temperatura relativamente fresca $\left(14-18^{\circ} \mathrm{C}\right)$ se han visto disminuidas notablemente, aumentando considerablemente el área de temperaturas altas. Para este estudio se ha considerado como islas de calor a aquellas áreas cuya temperatura sobrepasa $\operatorname{los} 26^{\circ} \mathrm{C}$ dando como resultado que estas se hallan en el cantón Rumiñahui en las áreas urbanas de Sangolquí y San Rafael además se encontraron pequeñas islas a lo largo de la parte rural, como se muestra en la Figura 2 donde se nota la diferencia de islas de calor del año 2001 al 2016. 

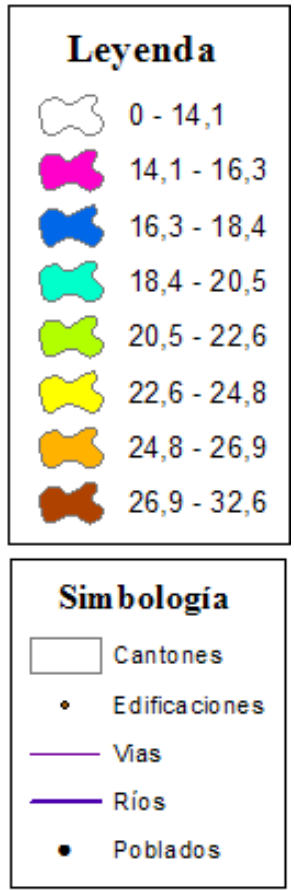

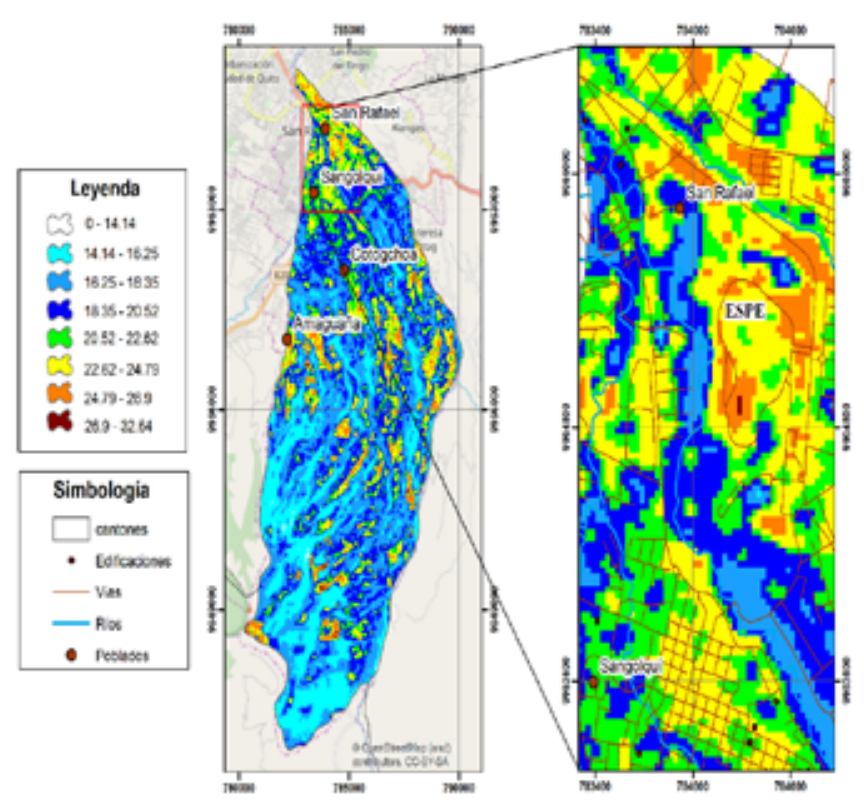

Figura 1. Mapa de temperatura (Imagen 2001)

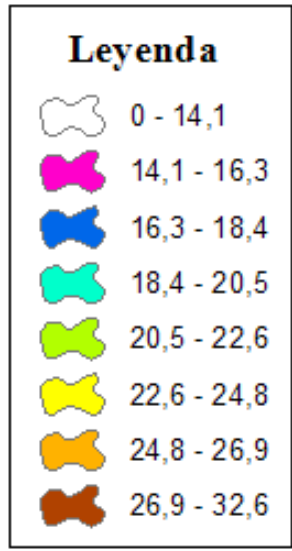

\begin{tabular}{|l|}
\hline \multicolumn{1}{|c|}{ Simbología } \\
\hline Cantones \\
- Edificaciones \\
\hline Vias \\
\hline Ríos \\
- Poblados
\end{tabular}

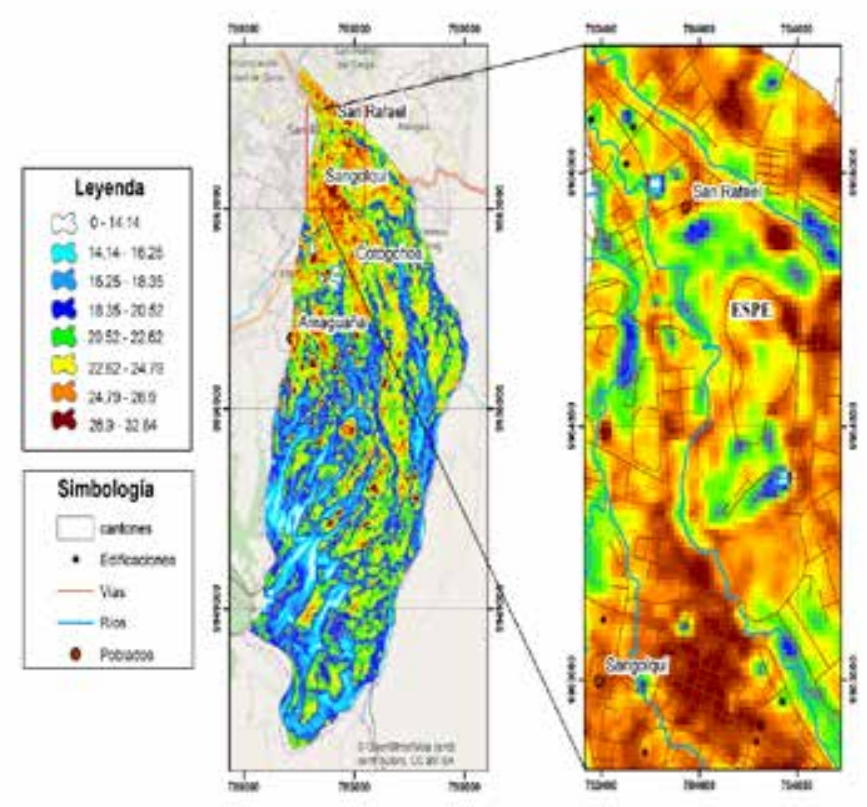

Figura 2. Mapa de temperatura (Imagen 2016) 
Las imágenes Landsat analizadas mostraron importantes diferencias en la temperatura superficial registrada en la zona urbana y periurbana, estas diferencias en el área que abarca cada rango de temperatura están cuantificadas mediante el porcentaje correspondiente en relación al área total del cantón Rumiñahui en la Tabla 3.

Tabla 3. Comparación de valores de área de temperatura en las dos fechas analizadas

\begin{tabular}{|c|c|c|}
\hline Temperatura $\left({ }^{\circ} \mathbf{C}\right)$ & \multicolumn{2}{|c|}{ Área (\%) } \\
\hline Rangos & Imagen 2001 & Imagen 2016 \\
\hline $0-14,14$ & 0,11 & 0,15 \\
\hline $14,1-16,3$ & 17,9 & 0,21 \\
\hline $16,3-18,4$ & 25,3 & 9,55 \\
\hline $18,4-20,5$ & 31,6 & 36,41 \\
\hline $20,5-22,6$ & 11,3 & 25,25 \\
\hline $22,6-24,8$ & 10,5 & 17,22 \\
\hline $24,8-26,9$ & 3,28 & 10,96 \\
\hline $26,9-32,6$ & 0,12 & 0,25 \\
\hline
\end{tabular}

Como puede verse en la Tabla 3, las áreas con una temperatura relativamente fresca que oscila entre 14 a $18^{\circ} \mathrm{C}$ se han visto disminuidas notablemente, aumentando considerablemente el área de temperaturas altas. Para este estudio se ha considerado como islas de calor a aquellas áreas cuya temperatura sobrepasa $\operatorname{los} 26^{\circ} \mathrm{C}$ dando como resultado que estas se encuentra en el área urbana de Sangolquí y San Rafael además se encontraron pequeñas islas a lo largo de la parte rural que puede ser resultado de edificaciones construidas en ese sector en las dos fechas analizadas como se distingue en la Figura 3.

(a)

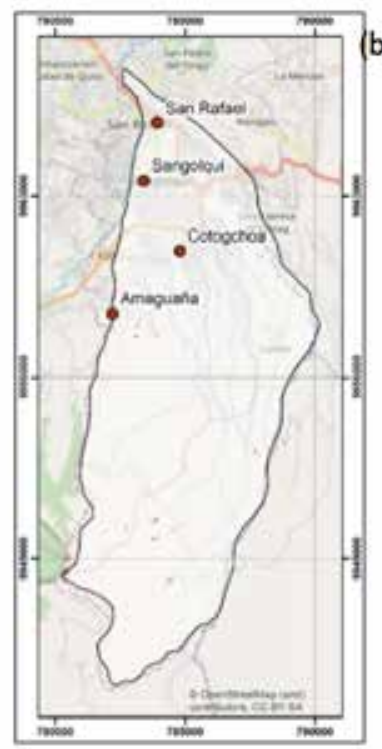

(b)

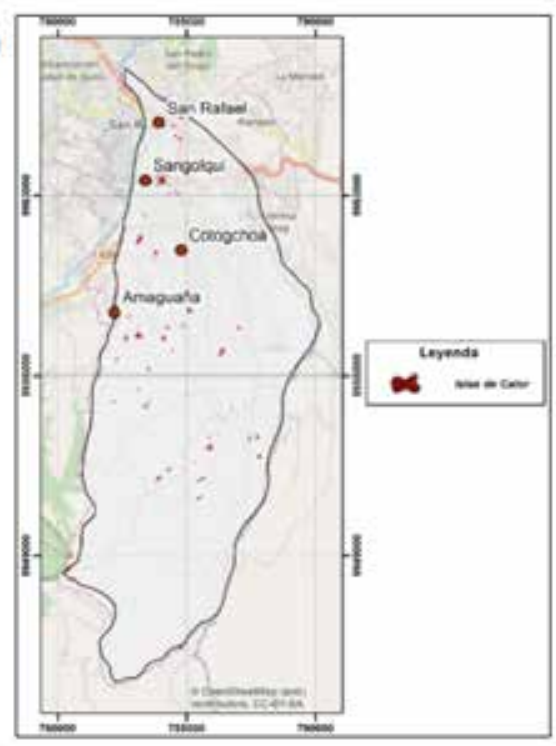

Figura 3. Islas de calor a lo largo del cantón Rumiñahui.: (a). Imagen 2001. (b) Imagen 2016 
De la misma manera se puede ver las diferencias en cuanto al valor de los índices: en los valores de NDVI es evidente que en la imagen correspondiente al 2001 se encuentra un gran porcentaje de vegetación saludable y vegetación en crecimiento, en las zonas urbanas (Sangolquí, Amaguaña, Cotogchoa) se observa que hay presencia de suelo desnudo y con poca vegetación como se ve en la Figura 4; en la imagen correspondiente al 2016 se encuentra mayores proporciones de suelo desnudo en la zona urbana. En cuanto a los valores de NDWI existe una mayor presencia de agua en la imagen tomada en el 2001 como se ve en la Figura 5 a comparación de la imagen del 2016 la cual presenta valores más bajos y en menor área, esto es consistente de acuerdo a los valores de vegetación mostrados en ambas imágenes. Por su parte, los valores de NDBI en la imagen de noviembre 2001 obtenidos, muestran en su gran mayoría, valores bajos como se ilustra en la Figura 6, lo que evidencia que no existe una gran presencia de construcciones mientras que en la imagen de octubre 2016 se muestra un gran incremento principalmente en lo que es el sector de Sangolquí y San Rafael.

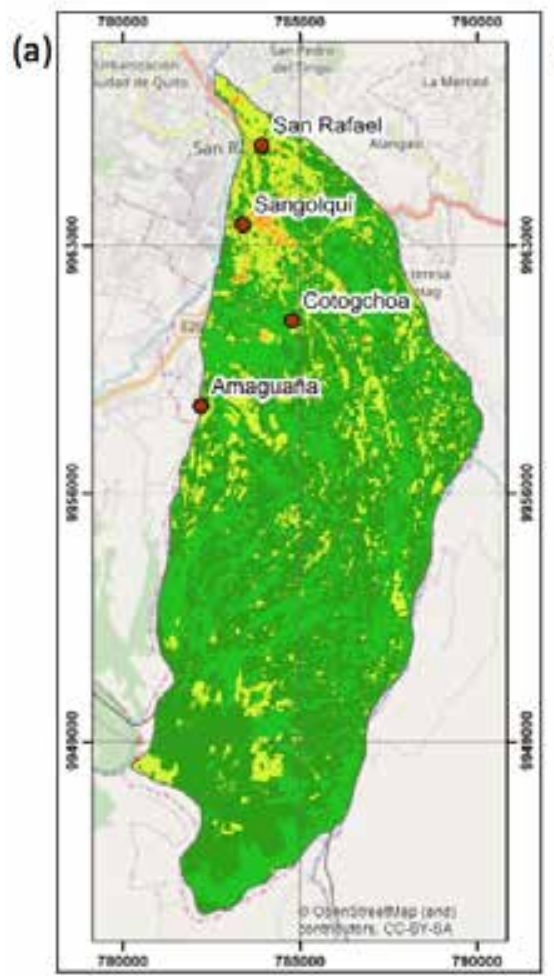

(b)
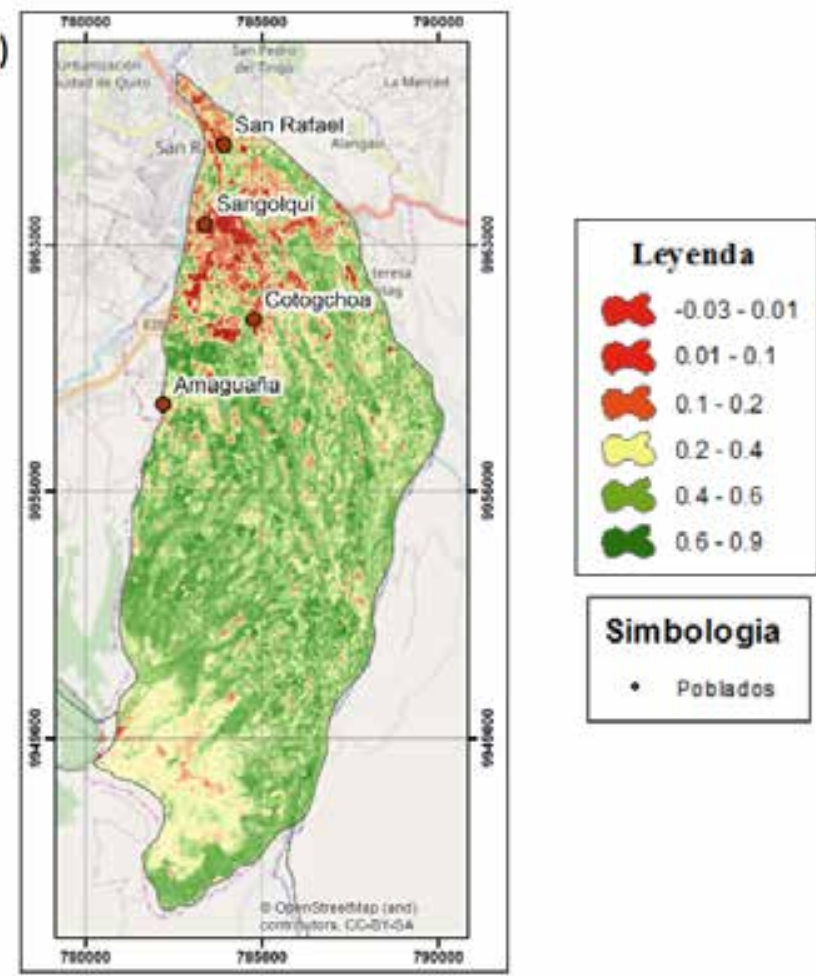

Simbologia

Figura 4. NDVI: (a). Imagen 2001. (b) Imagen 2016 
(a)

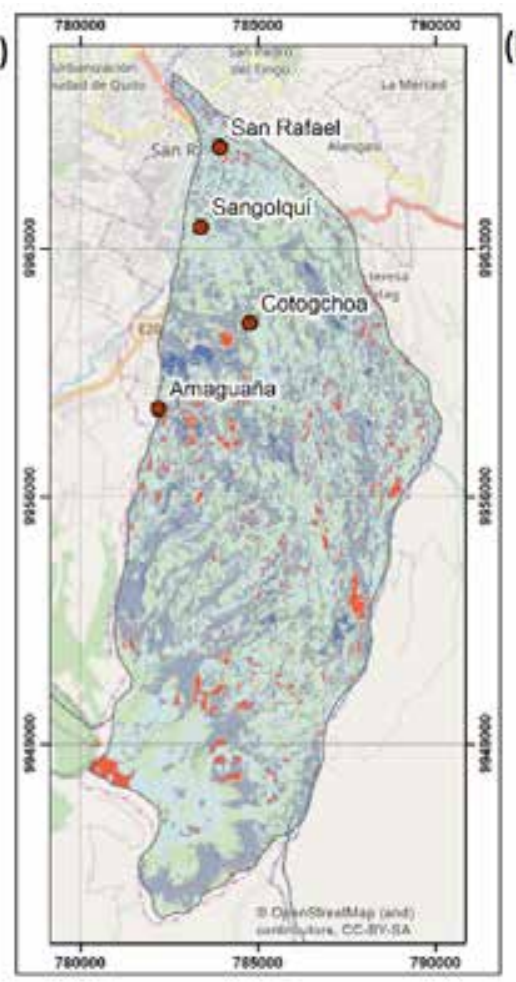

(b)

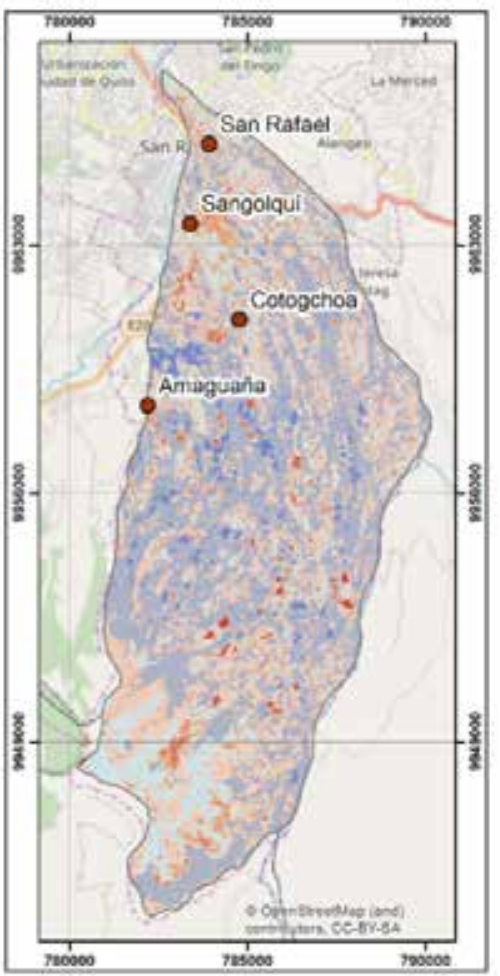

Figura 5. NDWI: (a). Imagen 2001. (b) Imagen 2016
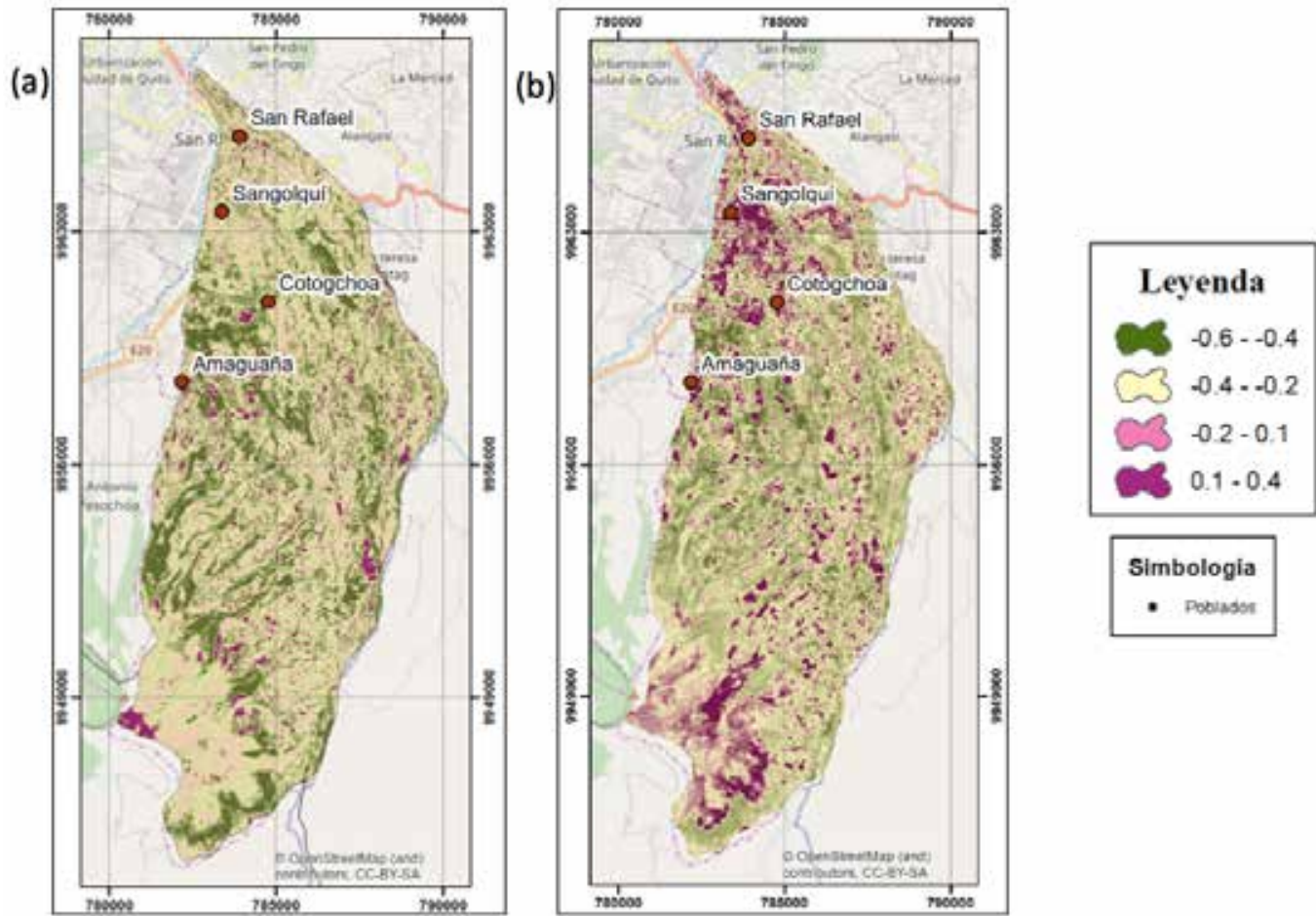

Simbologia

- Pobiasor

Figura 6. NDWI: (a). Imagen 2001. (b) Imagen 2016 


\section{ANÁLISIS ESTADÍSTICO}

Con los datos depurados se realizó el análisis de correlación entre las variables temperatura y los índices: NDVI, NDBI, NDWI y su fuerza de correlación se evaluó de acuerdo con los valores propuestos por (Montgomery , 2011) como se ve en la Tabla 4:

Tabla 4. Valores de correlación.

\begin{tabular}{|c|c|}
\hline Tipo de correlación & $\begin{array}{c}\text { Rango del valor del } \\
\text { coeficiente de correlación }\end{array}$ \\
\hline Correlación perfecta & $r=-1$ \\
\hline Correlación excelente & $-1<r<-0,9$ \\
\hline Correlación aceptable & $-0,9<r<-0,8$ \\
\hline Correlación regular & $-0,8<r<-0,6$ \\
\hline Correlación mínima & $-0,6<r<-0,3$ \\
\hline No hay correlación & $-0,3<r<0$ \\
\hline
\end{tabular}

En la figura 7 se observa que para la imagen del 2001 entre temperatura superficial y NDVI el coeficiente de correlación fue de $-0,95$, lo que indica una excelente correlación de tipo inversa entre las dos variables, en la figura 8 se muestra que la relación de temperatura superficial y NDWI la correlación arrojo un valor de $-0,67$ lo que indica que existe una correlación de tipo inversa regular; por último, en la figura 9 se observa que el coeficiente de correlación entre la temperatura superficial y el NDBI dio un valor de 0,68 , es decir una correlación directa regular, de esta manera la relación Temperatura - NDVI y NDWI es inversa mientras que con el NDBI es directa.

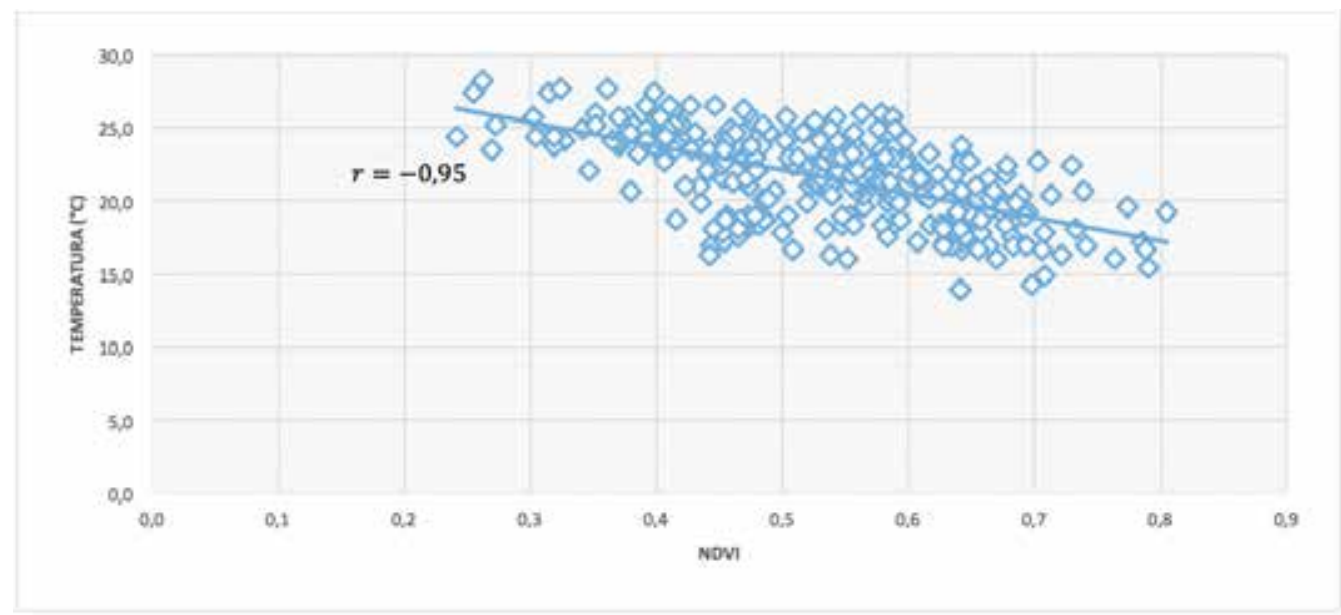

Figura 7. Diagrama de dispersión NDVI vs Temperatura (2001) 


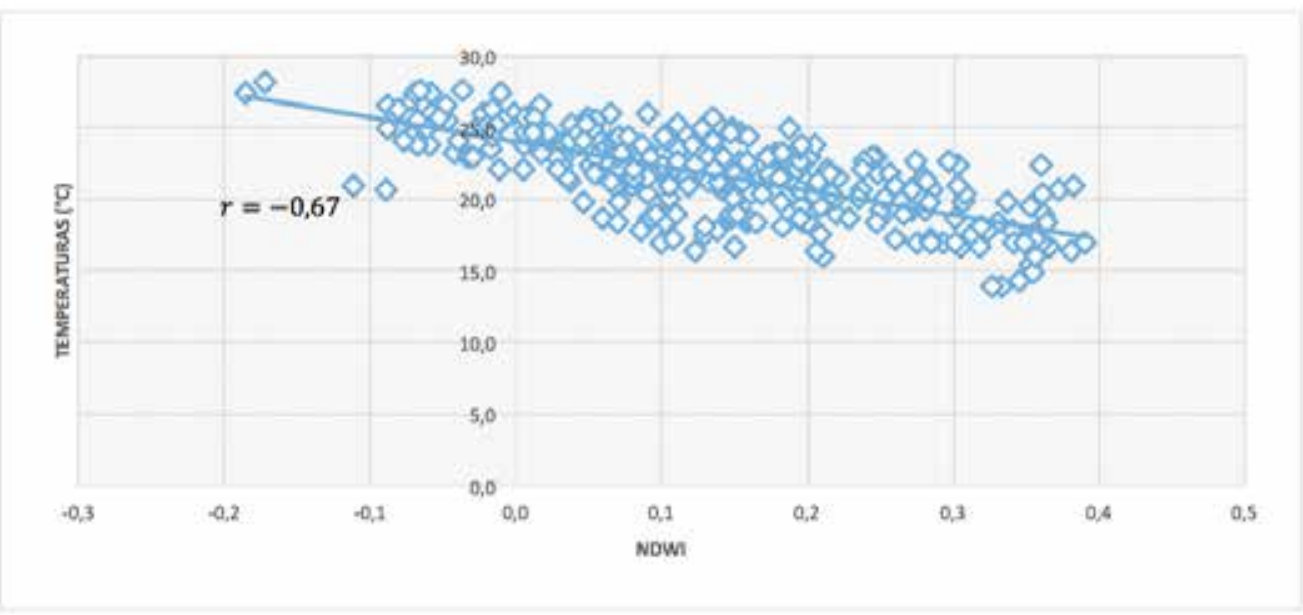

Figura 8. Diagrama de dispersión NDWI vs Temperatura (2001)

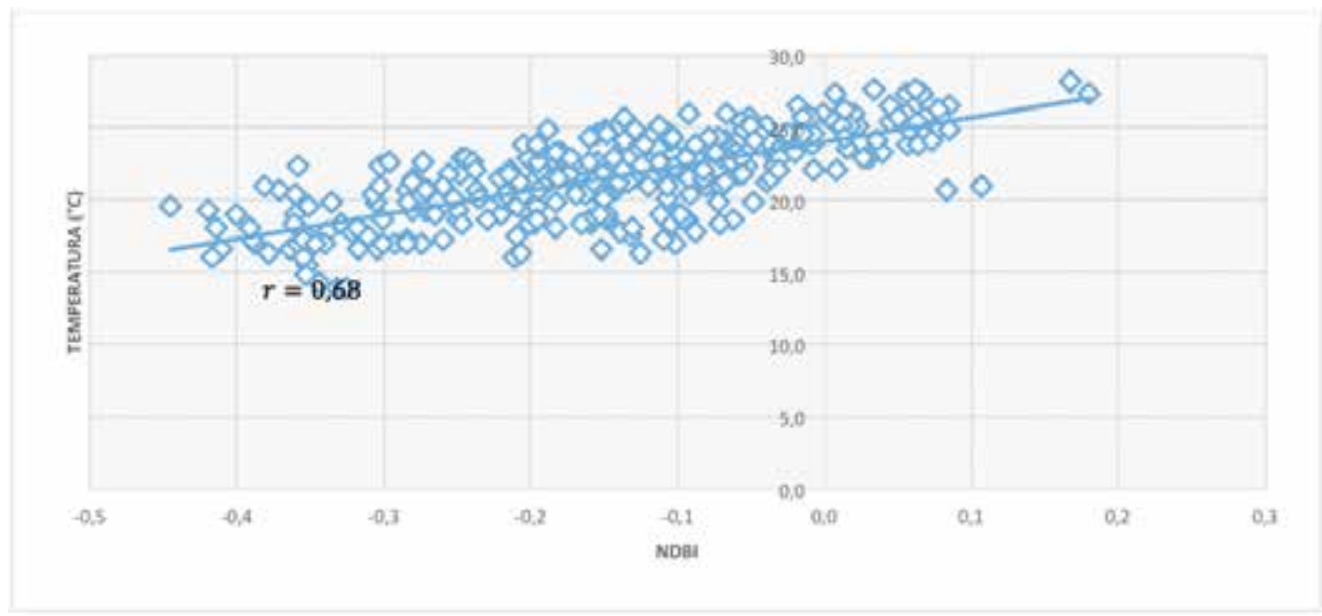

Figura 9. Diagrama de dispersión NDBI vs Temperatura (2001)

En cuanto a la imagen del 2016, como se muestra en la figura 10, la correlación se mostró notablemente más fuerte dando como resultado un coeficiente de -0,92 entre Temperatura y NDVI, lo que indica una excelente correlación de tipo inversa. En cuanto de NDWI se obtuvo un coeficiente de correlación inverso aceptable de $-0,85$ como se indica en la figura 11 y con respecto a NDBI se obtuvo un coeficiente de correlación directo aceptable de 0,87 como se muestra en la figura 12 . Las relaciones de proporcionalidad se mantienen iguales que en la imagen del 2001 entre las variables temperatura superficial e índices. 


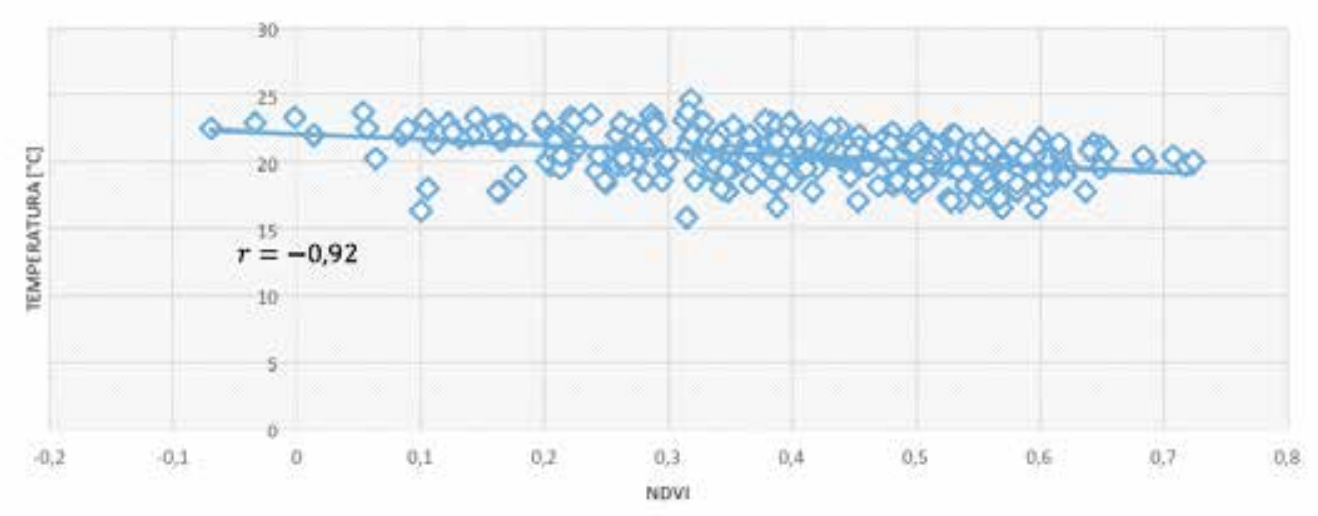

Figura 10. Diagrama de dispersión NDVI vs Temperatura (2016)

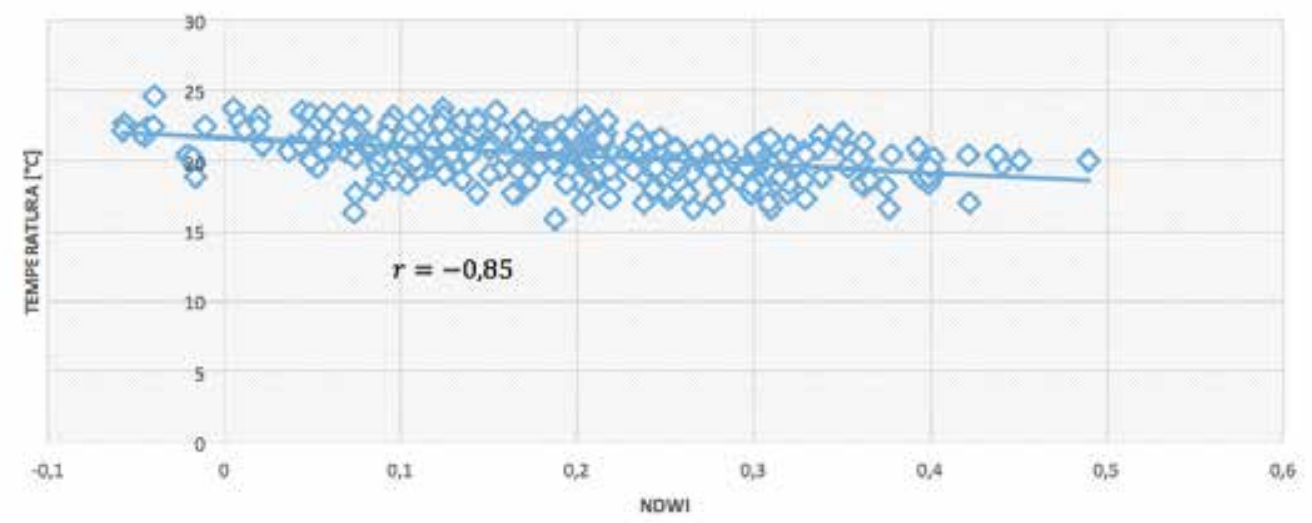

Figura 11. Diagrama de dispersión NDWI vs Temperatura (2016)

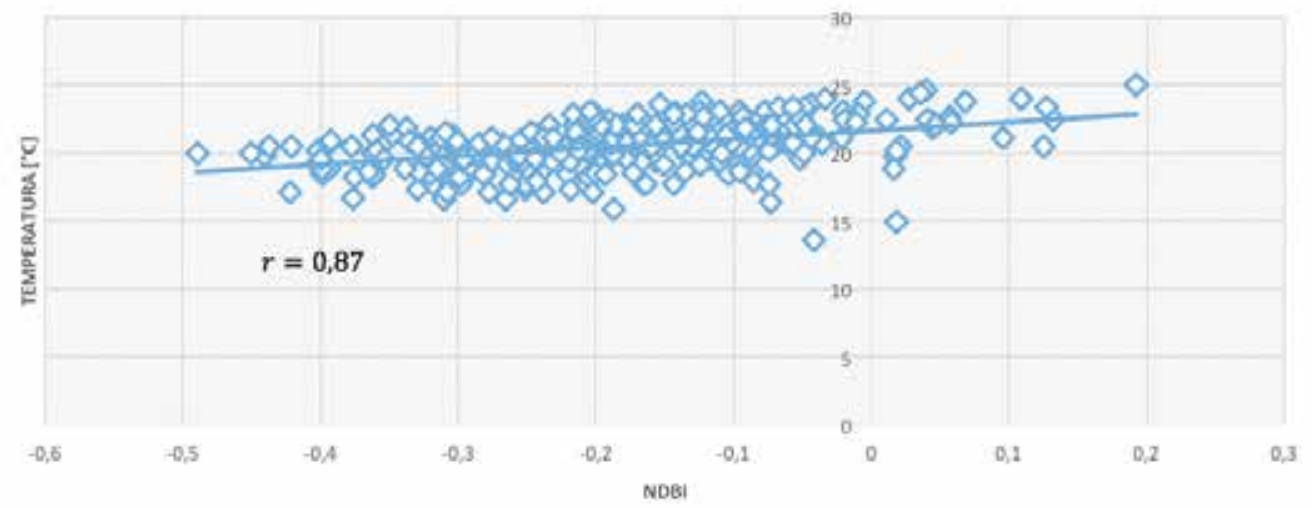

Figura 12. Diagrama de dispersión NDBI vs Temperatura (2016) 
Tabla 5. Valores de correlación obtenidos.

\begin{tabular}{|c|c|c|}
\hline Gráfico & Año & Correlación \\
\hline \multirow{2}{*}{ NDVI-TEMPERATURA } & 2001 & $-0,95$ \\
\cline { 2 - 3 } & 2016 & $-0,92$ \\
\hline \multirow{2}{*}{ NDWI-TEMPERATURA } & 2001 & $-0,67$ \\
\cline { 2 - 3 } & 2016 & $-0,85$ \\
\hline \multirow{2}{*}{ NDBI-TEMPERATURA } & 2001 & 0,68 \\
\cline { 2 - 3 } & 2016 & 0,87 \\
\hline
\end{tabular}

\section{DISCUSIÓN}

El porcentaje de islas de calor incrementó notablemente del 2001 al 2016, las temperaturas superficiales encontradas principalmente en los cascos urbanos tienen coherencia respecto a los valores de los diferentes índices, lo cual se evidencia con los valores de correlación encontrados entre las diferentes variables.

En la presente investigación se tuvo un coeficiente de correlación de 0,68 y 0,87 en cuanto a la TS - NDBI, esta es mayor a la encontrada en (Stamour \& Stella, 2014), la cual es de 0,45 , lo cual posiblemente tiende a mostrarse diferente debido a los espacios disponibles para asentamientos humanos de años pasados frente a los actuales, lamentablemente en la actualidad se opta por ocupar espacios propios de vegetación.

En cuanto a la TS - NDVI, el coeficiente de $-0,95$ y $-0,92$ es bastante similar al indicado en (Soberón \& Obregón, 2016), el cual es de 0,89 valores que reflejan lo mencionado en (Stamour \& Stella, 2014), que las temperaturas encontradas en las áreas verdes fueron relativamente bajas frente a otras coberturas diferentes a ésta.

En lugares donde existe abundante vegetación, igualmente podemos encontrar un alto índice de agua, lo cual se comprueba con el valor de 0,85 encontrado en la correlación TS-NDWI.

La similitud encontrada son diferentes estudios también evidencia la eficacia del uso de uso de sensores remotos para este tipo de estudios.

\section{CONCLUSIONES}

La distribución espacial de la temperatura superficial en el Cantón Rumiñahui, indica que existe presencia de islas de calor en los sectores de Sangolquí y San Rafael tanto en el año 2001 como en el 2016, cabe recalcar que el en año 2001 su porcentaje era $0,12 \%$ equivalente a 16,7 ha de área total del cantón mientras que en el 2016 aumento al 0,25\% en el 2016 lo que equivale a aproximadamente 34 ha del área total del cantón. 
El NDVI y NDWI muestran una correlación negativa (inversamente proporcional) con respecto a la temperatura, es decir, mientras la vegetación y agua presentes en el sector sean bajos, mayores temperaturas se encuentran en el mismo. Esta relación de proporcionalidad de muestra estable para las dos fechas tomadas, pero en cuanto al NDWI para el año 2016 la correlación se mostró más fuerte que en la fecha anterior.

El NDBI tiene relación directa con la temperatura superficial, el índice de construcciones es alto y la temperatura de la misma forma, también muestra como que se ha incrementado el dosel urbano a lo largo de los años además de indicar como se ha reducido la vegetación, es un claro ejemplo del cambio en el uso del suelo, ya que como se observa en el mapa en la parte rural del cantón se observa un mayor grado de vegetación en la imagen del 2001 a comparación del 2016. La correlación se mostró directa, es decir en las zonas donde se encuentra un índice elevado de construcciones también se encontró altas temperaturas.

Los resultados obtenidos tanto en temperatura superficial como en los diferentes índices contribuyen como referencia en la presencia de islas de calor principalmente en las zonas que se encuentren ubicadas en valles, o con características geográficas o climáticas similares a las del cantón Rumiñahui ubicado en el valle de Los Chillos.

\section{REFERENCIAS}

Barsi, J. (2005). Validation of a Web-Based Atmospheric Correction Tool for Single Thermal Band Instruments [en línea]. Earth Observing Systems, 5882, 2. Retrieved Marzo 2017, from https://atmcorr.gsfc.nasa.gov/Barsi_AtmCorr_SPIE05.pdf

Carreras, C., \& Martín, J. (1990). Modificaciones térmicas en las ciudades. Avances sobre la isla de calor en Barcelona. Documents d'Anàlisi Geogràfica, 17, 51-77.

Caselles, V. (2001). Desarrollo de una ecuación operativa para el cartografiado de emisividad. Teledetección, 2-5.

Chuvieco, E. (2010). Teledetección Ambiental (Tercera ed.). Barcelona, España: Planeta.

Córdova, K. (2011). Spatial Geotechnology applied to urban climate studies: Thermal analysis of urban surface and urban land use in the city of Caracas. Urban Climate News, 15-21.

EPA. (2009). Urban Heat Island Basics, Reducing Urban Heat Islands: Compendium of Strategies, Enviromental Protectio Agency, U.S, [Online]. Terra. Retrieved mayo 2017, from http:// www.epa.gov/hiri/resources/compendium.htm

Gao, B. (1996). A normalized difference water index for remote sensing of vegetation [en línea]. Remote Sensing of Enviroment, 58, 257-266. Retrieved Junio 2017, from http://www. sciencedirect.com/science/article/pii/S0034425796000673

Guillen Mena, V. (2016). Un acercamiento a caracterizar la Isla de Calor en Cuenca, Ecuador. CONAMA2016, 14. Retrieved from https://www.researchgate.net/publication/311509860 UN_ACERCAMIENTO_A_CARACTERIZAR_LA_ISLA_DE_CALOR_EN_ CUENCA_ECUADOR

IPCC. (2007). Cambio climático 2007; Informe de síntesis. Contribución de los grupos de trabajo I, II, III al cuarto informe de evaluacióndel grupo intergunamenta de expertos sobre el cambio climático. Ginebra, Suiza.

Marini, F. (2008). Análisis del estado de la vegetación en el área de influencia de la EEA [en 
línea]. Retrieved mayo 2017, from http:/inta.gob.ar/bordenave/contactos/autores/fabian/ indice_verde_area_INTA_bordenave.pdf

Montgomery, D. (2011). Probabilidad y Estadísticas aplicadas a la Ingeniería . New York : John Wiley.

Moreno, M. (1993). Estudio del clima urbano de Barcelona: la «isla de calor». (p. 193). Oikostau.

Moreno, M., \& Serra, J. (2016, Noviembre 25). El estudio de la isla de calor urbana en el ámbito mediterráneo: una revisión bibliográfica. GeoCritica, XXI(1.179). Retrieved Febrero 28, 2018, from http://www.ub.edu/geocrit/b3w-1179.pdf

NASA. (2017). Retrieved Julio 2017, from https://atmcorr.gsfc.nasa.gov/cgi-bin/atm_corr/atm_ corr.pl

Noro, M., \& Lazzarin, R. (2015). Urban heat island in Padua, Italy: simulation analysis and mitigation strategies. Urban Climate, 14, 187-196.

OMM. (2010). Statement on the status of the global climate in 2009. Ginebra, Suiza.

Palacios et al. (2017). La Forma Espacial de la Isla de Calor en la Ciudad de Guayaquil. INVESTIGATIO(9), 93.

Palacios, C. (2013). Caracterización del fenómenos "Isla Urbana de Calor" en la zona central de la ciudad de Guayaquil. Revista cientifica de la Universidad de Guayaquil, 10. Retrieved from https://issuu.com/editorialeduquilug/docs/edicion_116/11

Preciado, M., \& Aldana, A. (2011). Analisis de presencia de islas de calor en Santiago de Cali empleando técnicas de Teledetección. (p. 96). Manizales-Colombia: ISSN: 0123-9678.

SNI. (2010). Cifras generales 2010. 2. Retrieved Abril 2017, from http://app.sni.gob.ec/sni-link/ sni/Portal\%20SNI\%202014/FICHAS\%20F/1705_RUMI\%C3\%91AHUI_PICHINCHA. pdf

SNIA. (2016). SNIA. Retrieved Mayo 10, 2017, from http://dlibrary.snia.gub.uy/maproom/ Monitoreo_Agroclimatico/INDICES_VEGETACION/NDWI/

Soberón, V., \& Obregón, E. (2016). Identificación de islas de calor en la ciudad de Lima metropolitana utilizand imágenes del satélite Landsat 5TM., (p. 1). Lima-Perú. doi:http:// dx.doi.org/10.21704/ac.v77i1.475

Sosa , M. (2004). Manual de diseño para edificaciónes energeéticamente eficientes en el trópico. Universidad Central de Venezuela .

Stamour, A., \& Stella, M. (2014). Estimation of land surface temperature and urban patterns relationship for urban heat island studies., (p. 7). Grecce. Retrieved from https://www. researchgate.net/publication/260244679

Stewart, I. (2011). A systematic review and scientific critique of methodology in modern urban heat island literature. International Journal of Climatology, 31(2), 200-217.

Tumini, I. (2010). Estrategias para reducción del efecto isla de calor en los espacios urbanps. Estudio aplicado al caso de Madrid. Ponencia SB10 MAd, Edificación sostenible, Revitalización y Rehabilitación de Barrios., (p. 3).

United Nations. (2009). World urbanization propspects [en línea]. Retrieved Julio 2017, from http://esa.un.org/unpd/wup/index.htm 